Results At least one HR-HPV was identified in 264 HIV-negative men (37.6\%, 403 genotypes total) and 164 HIV-positive men $(70.4 \%$, 399 genotypes total) at enrollment. Among HIV-negative men, younger and unmarried men were more likely to have higher viral loads. HR-HPV genotypes with high viral load (grade:3-4) at enrollment were more likely to persist than HR-HPV genotypes with low viral load (grade:1-2) among HIV-negative (month 6: adjPRR = 1.80, 95\% CI: 1.31-2.47; month 12: adjPRR $=2.04$, 95\% CI: 1.39-3.01), and HIV-positive men (month 6: adjPRR = 1.33, 95\% CI: 1.06-1.67; month 12: adjPRR $=1.70,95 \% \mathrm{CI}: 1.16-2.50)$. Long-term persistence of HR-HPV was more frequent among HIV-positive men compared to HIV-negative men (month 24: adjPRR $=2.24,95 \% \mathrm{CI}$ : 1.46-3.45), and HR-HPV infections with low viral loads were detected more frequently among HIV-positive men at all follow-up visits (6 months: PRR $=1.81,95 \%$ CI: 1.17-2.97; 12 months: PRR $=1.43$, 95\% CI: $0.8-2.4 ; 24$ months: PRR $=2.9$, 95\% CI: $1.53-$ 5.53)

Conclusions HR-HPV genotypes with high viral load are more likely to persist among HIV-negative and HIV-positive men, though persistence was more common among HIV-positive men. The results may explain the association between high HR-HPV viral load and transmission to women and increased levels of HR-HPV persistence in HIV-positive men.

\section{P3.223 HPV GENOTYPE DISTRIBUTION IN HIV-POSITIVE AFRICAN WOMEN AND ASSOCIATIONS WITH HIGH GRADE HISTOLOGICAL LESIONS BY CD4+ COUNT}

doi:10.1136/sextrans-2013-051184.0680

${ }^{1} \mathrm{H}$ Kelly, ${ }^{2} \mathrm{~J}$ Ngou, ${ }^{3} \mathrm{~B}$ Sawadogo, ${ }^{4} \mathrm{~B}$ Muzah, ${ }^{1} \mathrm{C}$ Gilham, ${ }^{2} \mathrm{~N}$ Nagot, ${ }^{3} \mathrm{~N}$ Meda, ${ }^{4} \mathrm{~S}$ Delany, ${ }^{2} \mathrm{M}$ Segondy, ${ }^{1 P}$ Mayaud. 'London School of Hygiene and Tropical Medicine, London, UK; ${ }^{2}$ University of Montpellier 1 \& INSERM U1058, Montpellier, France; ${ }^{3}$ Centre de Recherche Internationale sur la Sante, Ouagadougou, Burkina Faso; ${ }^{4}$ Wits Reproductive Health and HIV Institute, Johannesburg, South Africa

Background To assess the prevalence, genotype distribution and risk factors for high-risk HPV among HIV-positive African women, and associations with cervical histological lesions.

Methods The HARP study enrolled HIV-positive women aged 25-50 in Burkina Faso (BF) and South Africa (SA). A stratified sampling strategy was used, with $2 / 3$ of women on ART. Cervical HPV genotyping was performed using InnoLipa. Four-quadrant cervical biopsies were obtained among women with abnormalities detected by at least one test or by colposcopy.

Results 628 and 624 women were enrolled in BF and SA, respectively. The distribution of CD4+ count (cells $/ \mu \mathrm{L})$ was similar in both sites: $68 \%$ with CD $4+\geq 350$ and $10 \%$ with CD $4+<200$. Prevalence of HR-HPV genotypes was $62 \%$ among women in BF and $78 \%$ in SA, and, overall, $67 \%, 73 \%$ and $84 \%$ among women with CD4 $+\geq 350,200-349$ and $<200$, respectively (Table). The 4 most common genotypes in BF were HPV52 (20\%), HPV51 (12\%), HPV35 (9\%), HPV66 (8\%); and in SA, HPV52 (24\%), HPV16 (15\%), HPV51 $(14 \%)$ and HPV35 (14\%). Multiple types were observed in $41 \%$ and $55 \%$ of HR-HPV-positive women in BF and SA, respectively; and increased with decreasing CD4 count $(46 \%, 52 \%$ and $63 \%$, respectively, P-trend $=0.004)$. HPV types 58,33 and 16 were most strongly associated with CIN2 $+(\mathrm{OR}=5.06, \mathrm{OR}=4.62, \mathrm{OR}=4.02)$ and types 16,35 and 58 were most strongly associated with CIN3+ $(\mathrm{OR}=4.59, \mathrm{OR}=3.36, \mathrm{OR}=2.96)$. Decreasing $\mathrm{CD} 4+$ count and younger age were associated with higher HR-HPV prevalence in both countries (Table). Multiple sex partners, smoking and lower income were also significantly associated with HR-HPV in SA.

Conclusions HR-HPV prevalence is high among HIV-positive women with genotype distribution similar in both countries. HRHPV prevalence is associated with young age and lower CD4+ count. Whilst HPV52 is the most prevalent type, HPV16 is most strongly associated with increasing lesion severity.
Abstract P3.223 Table 1 Table. Association of HR genotypes with site, CD4+ count and age in Burkina Faso and South Africa

\begin{tabular}{lll}
\hline & \multicolumn{2}{l}{ High risk HPV genotypes } \\
\hline & $\mathrm{n} / \mathrm{N}(\%)$ & $\mathrm{OR}(95 \% \mathrm{Cl})$ \\
Site & & $\mathrm{P}<0.001$ \\
Burkina Faso & $285 / 463(62 \%)$ & 1 \\
South Africa & $385 / 492(78 \%)$ & $2.24(1.69-2.99)$ \\
CD4+ count (cells/ $\mathrm{LL})$ & & $\mathrm{P}$-trend $=0.001$ \\
$<200$ & $76 / 90(84 \%)$ & 1 \\
$200-349$ & $151 / 206(73 \%)$ & $0.51(0.26-0.97)$ \\
$>=350$ & $442 / 658(67 \%)$ & $0.38(0.21-0.69)$ \\
Age group & & $\mathrm{P}$-trend $=0.002$ \\
$25-29$ & $140 / 178(79 \%)$ & 1 \\
$30-34$ & $184 / 254(72 \%)$ & $0.71(0.45-1.12)$ \\
$35-39$ & $158 / 237(67 \%)$ & $0.54(0.35-0.85)$ \\
$40-49$ & $188 / 286(66 \%)$ & $0.52(0.34-0.80)$ \\
\hline
\end{tabular}

\section{P3.224 EFFECT OF HERPES SIMPLEX VIRUS TYPE 2 (HSV-2) INFECTION ON PROGRESSION OF HIV INFECTION AMONG FEMALE SEX WORKERS IN BURKINA FASO}

doi:10.1136/sextrans-2013-051184.0681

II T Traore, ${ }^{2}$ Konate, ${ }^{1,2} \mathrm{~N}$ Meda, ${ }^{2} \mathrm{~W}$ Bazie, ${ }^{1} \mathrm{M}$ N Hema, ${ }^{2} \mathrm{~A}$ Kabore, ${ }^{2} \mathrm{D}$ Kania, ${ }^{3 \mathrm{P}}$ Mayaud, ${ }^{4,5} \mathrm{P}$ Van De Perre, ${ }^{4,5} \mathrm{~N}$ Nagot. 'University of Ouagadougou, Ouagadougou, Burkina Faso; ${ }^{2}$ Centre Muraz, Bobo-Dioulasso, Burkina Faso; ${ }^{3}$ London School of Hygiene and Tropical Medicine, London, UK; ${ }^{4}$ University of Montpellier, Montpellier, France; ${ }^{5}$ Unité INSERM U1058, Montpellier, France

Background The effect of HSV-2 on the natural history of HIV-1 remains unclear. Although trials have shown a modest but significant impact of HSV-2 suppression on HIV-1 disease progression, the sub-optimal antiviral efficacy of aciclovir and its potential antiretroviral effect have limited our ability to measure the true effect of HSV-2 on HIV-1 disease progression. This study aimed to assess the effect of untreated HSV-2 infection on the time to ART.

Methods From December 2003 to February 2012, HIV-1 infected female sex workers were enrolled in a prospective open cohort in Burkina Faso. At each 3-month follow-up visits, CD4 count and HIV-1 plasma viral load were done. Participants were offered care including ART and psychological support. Participants not on ART and having at least $350 \mathrm{CD} 4$ cells/ $\mu \mathrm{l}$ at enrolment (the current CD4 count threshold for ART initiation) were included in this analysis, which was censored at 36 months of follow-up when the assumption of proportional hazard was no longer met.

Results Overall, 164 co-infected women and 20 HIV-1 monoinfected women were enrolled in this study. At enrollment, the only difference between the two groups was a younger age of HIV-1 mono-infected women (median age 24 versus 31 years, $p<0.001$ ). In linear mixed models, the age-adjusted mean CD4 count at baseline (intercept) was significantly lower among HSV-2 positive women (-211 cell/ $\mu \mathrm{L}, \mathrm{p}<0.001)$, but no difference in baseline CD4-adjusted plasma viral load was observed. During follow-up, 3 out 20 HIV-1 mono-infected women initiated ART versus 52 out of $164 \mathrm{HSV}-2$ co-infected women. After adjustment for baseline CD4 count and age, HSV-2 infected women were still much more likely to initiate ART over 36 months (HR = 4.6, CI 95\%: 1.04-20.5, p = 0.04).

Conclusion HIV-1 disease progression, as assessed by time to ART eligibility, was much accelerated for women co-infected with HSV-2.

\section{P3.225 HSV-2 SEROPREVALENCE AMONG CURRENT INJECTION DRUG USERS IN ESTONIA}

doi:10.1136/sextrans-2013-051184.0682 